Revista Calidad en la Educación Superior

Programa de Autoevaluación Académica

Universidad Estatal a Distancia, Costa Rica

ISSN 1659-4703

revistacalidad@uned.ac.cr

\title{
PRINCIPIOS DE LA RETROALIMENTACIÓN DESDE EL DIÁLOGO DIDÁCTICO MEDIADO
}

FEEDBACK PRINCIPLES FROM THE MEDIATED DIDACTIC DIALOGUE APPROACH

\author{
Natalia Salas Quirós ${ }^{1}$ \\ nsalas@uned.ac.cr \\ Universidad Estatal a Distancia, Costa Rica
}

\author{
Volumen 7, Número 1 \\ Mayo 2016 \\ pp. 77 - 99
}

Recibido: 10 octubre del 2015

Aprobado: 23 de febrero del 2016

\footnotetext{
${ }^{1}$ Bachiller en Educación Preescolar, Licenciada en Administración Educativa y Master en Planificación Curricular por la Universidad de Costa Rica. Asesora curricular de procesos de diseño o rediseño de planes de estudio y asignaturas en el Programa de Apoyo Curricular y Evaluación de los Aprendizajes de la Universidad Estatal a Distancia, Costa Rica (UNED). Correo electrónico: nsalas@uned.ac.cr
} 


\section{Resumen}

La educación a distancia comprende un proceso esencialmente comunicativo, que se caracteriza por tener una finalidad educativa a través de diferentes medios y materiales didácticos; dentro del cual la retroalimentación como parte de la evaluación formativa de los aprendizajes, es un elemento fundamental para favorecer en el estudiantado la autorregulación y autonomía. Así, el presente artículo corresponde a una revisión bibliográfica cuyo objetivo fue establecer los principios de la retroalimentación desde el diálogo didáctico mediado como estrategia para el favorecimiento de aprendizajes significativos y el desarrollo de la capacidad de aprender a aprender en el estudiantado de la educación superior a distancia; en el que se determina como principios básicos la retroalimentación a tiempo, con interacción multidireccional y que dé respuesta a tres preguntas claves: ¿A dónde voy? ¿Cómo voy? ¿Qué sigue?

Palabras clave: retroalimentación; proalimentación; educación superior a distancia; diálogo didáctico mediado; autorregulación del aprendizaje

\section{Abstract}

The distance education includes an essentially communicative process, which is characterized by having an educational aim through different media and materials; within which the feedback as part of the formative evaluation of learning, is a fundamental element in favor of selfregulation and autonomy in the student body. Thus, this article corresponds to a bibliographical review whose purpose was to establish the principles of feedback from didactic dialogue mediated as a strategy for the promotion of meaningful learning and the development of the ability to learn to learn in higher distance education students; on that is determined, as basic principles, the feedback in time, with multidirectional interaction and that give answers to three key questions: Where am I going? How do I go? What's next?

Keywords: feedback; feed forward; higher education at a distance;mediated didactic dialogue; self-regulation of learning

\section{Justificación}

La educación a distancia $(\mathrm{EaD})$ es una modalidad educativa que se encuentra basada en el diálogo didáctico mediado, es decir se vale de diversos medios, recursos y materiales para acercar al estudiantado al objeto de conocimiento, a través de diversas formas de interacción que permita establecer una relación dialéctica entre objeto, sujeto y contexto. 
En la actualidad, se cuenta con la riqueza de formas de comunicación didáctica de las primeras generaciones de medios y materiales didácticos de la educación a distancia como lo son el libro, la radio, los documentales, entre otros, junto con las nuevas herramientas tecnológicas de la Web 2.0, con las cuales dicho diálogo didáctico se concreta desde una interacción en múltiples vías (García, 2014); es decir, de cada estudiante con el profesorado, con sus pares, con los recursos y materiales didácticos, así como con el contexto; ya sea de forma sincrónica o anacrónica.

Al respecto, Moreno (2012) comenta

El uso de las TIC y el concepto de comunicación a distancia con usos pedagógicos crean un nuevo ambiente educativo. Al incorporarse las TIC (en especial las redes digitales), se posibilita aún más una acción pedagógica centrada en el estudiante, flexible, abierto, pero no necesariamente autodidacta, que fomenta la autogestión (...) (p.12)

En este sentido, es importante tener claro que la finalidad de interacción en los procesos educativos es propiciar no sólo la construcción de conocimientos sino también el desarrollo de la capacidad de autorregulación, que conlleva al desarrollo de la autonomía, la autodisciplina del estudiantado a través de procesos de metacognición (Lozano y Tamez, 2014); lo cual significaría que la persona que ingrese a estudiar en un sistema a distancia requiere de estas capacidades no sólo para tener un buen desempeño en sus estudios sino que además en su vida personal y profesional, donde los diferentes medios, recursos y materiales didácticos juegan un importante papel para mediar el aprendizaje.

De este modo, como parte de los desafíos de la educación superior a distancia, se encuentra el desarrollo de un modelo de evaluación de los aprendizajes que sea congruente con las tendencias y necesidades actuales de la sociedad del conocimiento en cuanto al perfil socioprofesional de las personas que se gradúen desde los diferentes campos de acción, entre las cuales están la capacidad de resolución de problemas, la autonomía, autodisciplina, el liderazgo y la iniciativa. 
Dicho modelo responde, según Brown en Brown y Glasner (2010) a factores tales como: el replanteamiento del curriculum institucional, diversidad de perfiles de la población estudiantil que ingresa a la universidad, así como el tiempo destinado para los trabajos evaluativos y presiones al profesorado para brindar la retroalimentación. A estos se agrega las nuevas formas de acceder a la información, lo que aprende el estudiantado y cómo lo aprende (Rodríguez e lbarra, 2011).

En este contexto, la docencia en la educación a distancia requiere visualizarse desde un rol de facilitación y orientación del proceso de aprendizaje, a fin de que el estudiantado adquiera gradualmente la capacidad de aprender a aprender, a partir de la interacción con los demás y el entorno; lo cual implica necesariamente procesos donde la evaluación de los aprendizajes tiene una visión formativa y formadora.

No obstante, en las prácticas educativas en la educación superior aún tiene un especial interés la certificación del nivel de conocimientos, por encima del proceso de construcción de conocimientos que ha llevado a cabo cada estudiante, a sabidas cuentas que no necesariamente una calificación refleja lo que realmente el estudiantado ha aprendido.

Consecuentemente, es común que la retroalimentación se centre en el producto (entiéndase la resolución de una prueba escrita, un informe, u otro tipo de trabajos) mediante la asignación de un número, en ocasiones acompañados por comentarios; esto hace que la información que se proporciona no llegue en el momento oportuno o que no sea significativa para que cada estudiante pueda mejorar.

Al respecto, diversos autores como: Lozano y Támez, 2014; Wiggins, 2012; Gibbs, en Brown y Glasner, 2010; Sanmartí, 2007, Hattie y Timperley, 2007 señalan como principales problemáticas del uso de la retroalimentación la falta de continuidad por parte del profesorado a cada estudiante, lo inoportuno y la poca personalización con que se da la información de retorno, lo cual redunda en el desinterés por parte del estudiantado por la retroalimentación que se les brinda. 
En esta perspectiva, se limita la retroalimentación a la que brinda el profesorado, que si bien es necesaria, no es la única posible. Desde la perspectiva en que se visualiza en la actualidad la educación superior a distancia, la interacción entre los agentes que intervienen en el proceso educativo es preponderante.

Adicionalmente, en los escritos acerca de la retroalimentación en educación a distancia es más frecuente encontrar propuestas basadas en ambientes virtuales, donde prima la interacción directa del profesorado con el grupo de estudiantes. Surge, entonces, la pregunta, ¿es posible la retroalimentación solamente haciendo uso de las tecnologías de la información y comunicación? ¿Qué sucede en el caso de los materiales didácticos como el libro, los audiovisuales $u$ otros propios de las primeras generaciones de la educación a distancia que aún siguen siendo utilizados?

Por tal motivo, esta revisión bibliográfica tiene como objetivo establecer los principios de la retroalimentación desde el diálogo didáctico mediado como estrategia para el favorecimiento de aprendizajes significativos y el desarrollo de la capacidad de aprender a aprender en el estudiantado de la educación superior a distancia.

\section{Metodología}

Este estudio corresponde a una investigación de tipo revisión bibliográfica descriptiva, la cual surge dentro del proyecto investigativo "Evaluar para aprender en la UNED" del Programa de Apoyo Curricular y Evaluación de los Aprendizajes (PACE) de la UNED de Costa Rica, el mismo se encuentra en proceso de ejecución.

Para dicha revisión se realizó una búsqueda de información bibliográfica en las bases de datos de la UNED de Costa Rica, tanto en formato físico como digital y en el buscador de Google Scholar; así como en sitios web de asociaciones o redes de investigación sobre el tema. También, se hizo uso de la herramienta Zotero para la recopilación de los diferentes documentos encontrados en la Web. La revisión se realizó entre los meses de mayo y julio de 
2015 con los descriptores: retroalimentación, feedback, evaluación formativa, autorregulación, educación a distancia, educación superior, diálogo didáctico mediado, comunicación didáctica.

\section{Desarrollo y discusión}

\section{La educación a distancia (EaD) un acto comunicativo.}

Desde el surgimiento de la educación a distancia han estado presentes las formas de establecer un proceso comunicativo con el estudiantado, teniendo en cuenta dos aspectos elementales; a saber, por un lado que "toda intención educativa implica un proceso de comunicación que tiene por objeto propiciar la comprensión de algo y/o la solución de problemas a través de la reflexión" (Rodríguez, 2007, pp-39-40); y por otro, la separación (temporal o espacial, o ambas) entre el profesorado y el estudiantado, como una de las características esenciales de esta modalidad educativa (García, 2014).

En este sentido, desde la enseñanza por correspondencia donde se enviaba a cada estudiante material escrito para su autoformación hasta el uso de las tecnologías de información y comunicación, se ha visto la necesidad de poder establecer un sistema óptimo que permita a la población estudiantil aprender a través de distintas formas y canales de comunicación. Sin embargo, el para qué y el cómo de esa comunicación ha estado influenciado por el sustento y las prácticas pedagógicas que se desarrollan en los procesos educativos a distancia tanto en el pasado como en la actualidad.

De esta manera, Anderson y Dron (2011) distinguen tres generaciones pedagógicas: la del conductista-cognitivo, la del socio-constructivismo y la del conectivismo. La primera de ellas se distingue por actividades en las que interesa únicamente que el estudiante observara y leyera materiales escritos, programas de radio o televisión o aquellos donde la comunicación se hacía uno a uno; es decir, de cada estudiante con el profesorado; mientras que la evaluación de los aprendizajes se centraba en la memorización pues el interés radica principalmente en los contenidos y el conocimiento. 
En el caso del socio constructivismo, las actividades buscan la discusión y el desarrollo de propuestas como solución a diversas problemáticas, así se introducen herramientas tecnológicas audiovisuales y de la Web que permitan la comunicación en múltiples direcciones; es decir, de cada estudiante con el profesorado, con sus pares y con los materiales a disposición, en la que la evaluación de los aprendizajes busca el desarrollo de capacidades superiores de conocimiento. Aquí se ubica una generación pedagógica que coloca al estudiantado como centro del proceso educativo.

Más recientemente, surge el conectivismo que tiende al desarrollo de actividades de exploración, conexión, creación y evaluación, donde las herramientas de la Web 2.0 ocupan un lugar preponderante para la creación de redes de comunicación y comunidades de aprendizaje; donde la evaluación de los aprendizajes se centra en la creación de artefactos o en productos de aprendizaje.

De acuerdo con Anderson y Dron (2011) cada una de estas generaciones pedagógicas, al igual que las generaciones tecnológicas no se pueden catalogar en un espacio de tiempo específico, pues desde su surgimiento se encuentran presentes en las diferentes propuestas de educación a distancia, ya sea su propuesta curricular como en las prácticas que se llevan a cabo.

Ciertamente, a partir de las demandas, tendencias y necesidades actuales de la sociedad es necesario promover, en el estudiantado de educación superior, el desarrollo de capacidades que van más allá del manejo de lo propio del campo profesional; esto es el trabajo en equipo, la resolución de problemas, la proactividad, autonomía, autorregulación, iniciativa, criticidad, creatividad entre otras (Medel-Añonuevo, Ohsako y Mauch, 2001; CSUCA, 2013; García, 2014).

En este sentido, pareciera ser que los postulados socioconstructivistas y el conectivismo adquieren un papel importante en respuesta a dichas demandas y tendencias, por estar centrados en los procesos de construcción de conocimiento, favorecer la interacción como uno 
de los elementos esenciales del aprendizaje y darle protagonismo a la persona estudiante. Al respecto, Gutiérrez y Prieto desde el año 1999 señalaron que la EaD debería caracterizarse por los siguientes aspectos:

(...) ser participativa a pesar de la distancia, partir de la realidad y fundamentarse en la práctica social del estudiante, promover en los agentes del proceso actitudes críticas y recreativas, abrir caminos a la expresión y a la comunicación, promover procesos y no sólo obtener productos, fundamentarse en la producción de conocimientos, ser lúdica, placentera y bella, desarrollar una actitud investigativa (Gutiérrez y Prieto, 1999, p.45)

Esto significa que el papel docente debe ser el de guía, facilitador y mediador de los procesos de construcción de conocimientos que realice el estudiantado, pues más allá de dominar un conjunto de contenidos interesa el desarrollo de la capacidad de aprender a aprender.

Así, los procesos educativos deberán ser planteados desde la persona estudiante como protagonista, dinámica y activa en su construcción de conocimientos, donde los espacios comunicativos que se generen deberán procurar un diálogo didáctico mediado, en el cual se establezca un intercambio entre profesorado y estudiantado que permita orientar el proceso de aprendizaje, a partir de las múltiples interacciones con el objeto de conocimiento, los pares, el entorno y demás agentes educativos.

Si bien es cierto, este diálogo y la interacción es una condición fundamental en la EaD, según González (1988, fue Holmberg en 1985 quien presenta un modelo de educación basado en lo que él llamó la conversación didáctica guiada, la cual se basa en una comunicación real o simulada, esta última referida a la interacción que se debe dar desde los materiales y recursos que se brinden al estudiantado.

A finales de 1980 e inicio del año 2000, según Simonson, Smaldino, Albright y Zvacek (2009), Garrison propuso el modelo de EaD de comunicación bidireccional, desde el cual asegura que "la calidad y la integridad del proceso educativo depende en la comunicación en dos vías" (p.80). Más adelante, autores como Anderson (2008) amplían este modelo de EaD desde una 
propuesta de seis formas de interacción que incluyen al estudiantado con sus pares, con el profesorado, con el contenido, con el entorno, del profesorado entre sí y del profesorado con el contenido, a partir de un ambiente de aprendizaje colaborativo con un estudiantado autónomo.

Más actualmente, García (2014) retoma los aportes de las teorías y modelos de la EaD y propone el diálogo didáctico mediado (DDM), el cual se desarrolla a partir de lo que él llama los cuatro componentes esenciales de la EaD: "docencia (institución), aprendizaje (alumnos), materiales (contenidos) y comunicación (vías y canales)" (p. 94), con una dimensión social referida al intercambio entre quienes participan del acto educativo, una dimensión pedagógica que orienta a los propósitos de aprendizaje y una dimensión tecnológica que hace uso de diferentes medios y materiales como vías o canales para facilitar el proceso de construcción de conocimientos.

Así, el autor afirma:

Este modelo teórico del DDM basa su propuesta en la comunicación a través de los medios, que cuando se trata de los materiales, descansa en el autoestudio y cuando se trata de las vías de comunicación, en la interacción vertical y horizontal” (p.94)

Entonces, desde el DDM se pueden encontrar varios tipos de diálogos en la comunicación educativa: simulados 0 reales, sincrónicos 0 anacrónicos, unidireccionales 0 multidireccionales, verticales $u$ horizontales, estructurados o flexibles; los cuales pueden presentarse en combinaciones diversas entre sí; especialmente, con la incorporación de las herramientas propias de las tecnologías de la información y la comunicación que abre un amplio panorama de posibilidades en la educación a distancia en términos de interacción y de multidireccionalidad en la comunicación; lo que conlleva a que en momentos del proceso educativo el estudiantado tome la iniciativa para iniciar el diálogo.

Tal y como señala Simonson, Hudgings y Orellana (2009) este diálogo debe permitir al estudiantado tomar en consideración diferentes puntos de vista, buscar soluciones y 
reflexionar sobre el proceso de aprendizaje con y desde los demás, lo que significa generar habilidades de reflexión, capacidad crítica y resolución de problemáticas, así como de autorregulación, que son esenciales para las exigencias de la sociedad actual.

\section{El proceso de retroalimentación para la construcción del conocimiento desde el DDM.}

Ahora bien, en la dinámica del proceso de construcción de conocimientos, dentro de esa mediación que se realiza desde el DDM, se encuentra el proceso de retroalimentación como un elemento esencial, desde el cual se pretende brindar una orientación oportuna, válida y útil al estudiantado sobre su aprendizaje, el cual tiene gran importancia en el desarrollo de la autonomía del estudiante (Ros I Solé y Truman citados en Holmberg, Shelley y White, 2005; Ferguson, 2011).

Para la retroalimentación se encuentran diversos significados, de los cuales se extraen algunas posiciones más relevantes al respecto, según la revisión bibliográfica realizada.

Así, para Ros I Solé y Truman citados en (Holmberg, Shelley y White, 2005) la retroalimentación consiste de tres intervenciones por parte del profesorado: "la corrección de los errores en estudiantes, la guía provista de errores en particular o problemas y los comentarios con consejos generales para el mejoramiento del desempeño" (p.73), que en el caso de la educación a distancia debe tener como finalidad promover el desarrollo de estrategias de autorregulación.

En el caso de Hattie y Temperley (2007) la retroalimentación consiste en "la información provista por un agente (por ejemplo profesor, par, libro, pariente, sí mismo, la experiencia) de acuerdo con aspectos de desempeño o comprensión del otro" (p. 81) (traducido). En el año 2014, Lozano y Tamez retoman esta definición e indican que la retroalimentación es la "acción en la que el profesor proporciona al estudiante información enfocada a mejorar el producto 0 proceso académico" (201) 
Sanmartí (2007), por su parte menciona que la retroalimentación es el proceso de gradualidad analógica que devuelve información procesada para consolidar los conocimientos adquiridos y poder ir más allá (p.6). Mientras que Wiggins (2012) afirma que esta es "básicamente la información acerca de cómo estamos haciendo nuestro esfuerzo para alcanzar una meta" (p.11) (traducido)

En el caso de la educación a distancia Simonson (2009) afirma:

La respuesta de los aprendientes que reciben el mensaje es llamada retroalimentación. La retroalimentación permite al emisor y al receptor, al profesor y al estudiante, determinar si el mensaje fue comprendido correctamente. La retroalimentación en los sistemas de educación a distancia está frecuentemente referida a la interacción. La retroalimentación permite a aquellos involucrados en la comunicación en un sistema de educación a distancia evaluar el proceso (p.91) (traducido)

Desde estas posturas, se puede encontrar en común que existe una persona o personas que brindan una información a otra acerca de la forma en que se está desempeñando de acuerdo con un proceso de aprendizaje o con una meta u objetivo establecido, lo que implica una interacción entre las personas involucradas (ver figura 1). Esto significa que la retroalimentación en sí es un proceso de intercambio de información entre docente-estudiante, estudiante-docente, estudiante-sus pares, estudiante-materiales didácticos; es decir, requiere de un papel mucho más activo del estudiantado como protagonista de su proceso de aprendizaje; así como el replanteamiento del rol de la docencia sobre todo tomando en cuenta si se realiza desde un modelo de educación a distancia, donde interviene el diálogo didáctico simulado y el diálogo didáctico sincrónico o el anacrónico. 
Figura 1. Elementos esenciales que intervienen en la retroalimentación

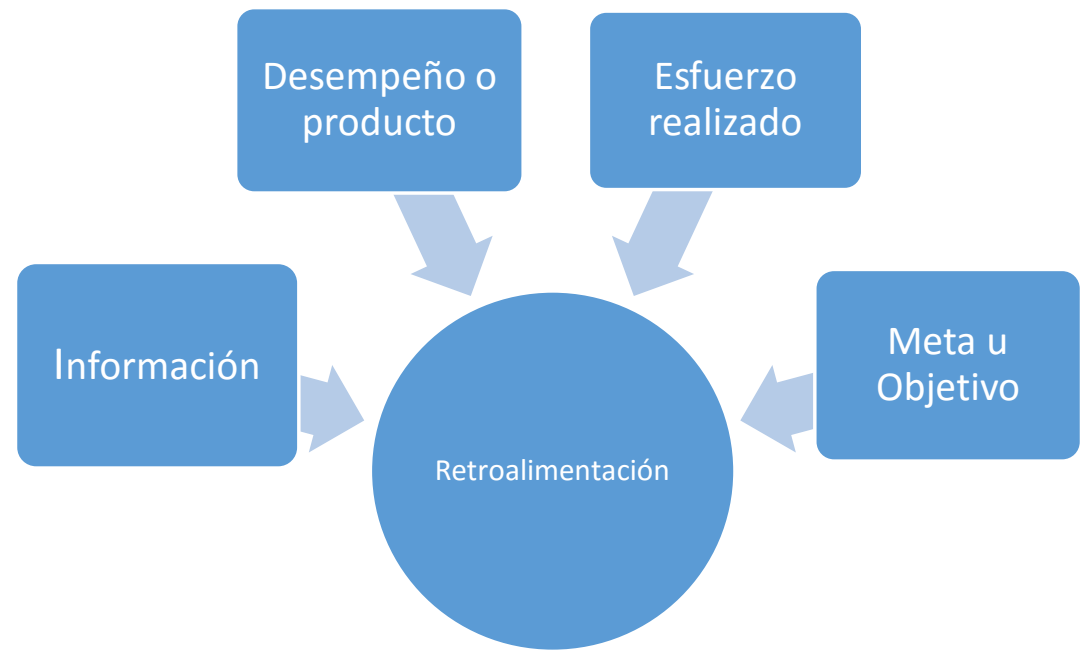

Fuente: Elaboración propia

No obstante, el tipo de información que se brinda, cuándo y cómo se ofrece desde la EaD, son aspectos necesarios por considerar, pues pueden llegar a ser determinantes en la consecución del aprendizaje esperado.

De acuerdo con la investigación realizada por Evans (2013) el papel de la retroalimentación "depende del ambiente de aprendizaje, las necesidades de quien aprende, el propósito de la tarea y el paradigma en que se base la retroalimentación" (p.71) Así, menciona el cognitivismo y el socio-constructivismo como dos paradigmas desde los cuales se ha abordado la retroalimentación, los cuales indica la autora, se pueden complementar entre sí.

Al igual que en la forma en que se concibe la educación a distancia, la conceptualización de la retroalimentación debe partir del enfoque que sustente la práctica educativa. Es así que desde el cognitivismo la retroalimentación se asume desde un punto de vista correctivo, donde el profesorado (que es quien sabe) brinda la información al estudiante que no da información de retorno (2013). 
Mientras que en el socio-constructivismo se basa en el diálogo y la participación del estudiantado para el desarrollo de la consciencia acerca de lo que se ha aprendido y qué se requiere mejorar a partir de dar sugerencias y comentarios que permitan a cada estudiante autoevaluarse y coevaluar (2013); lo cual implica la participación de sus pares en el proceso de retroalimentar la construcción de conocimientos, con miras a construir una comunidad de aprendizaje.

En este sentido, se parte del supuesto de que la retroalimentación, como uno de los componentes de la evaluación formativa, deberá orientarse a la regulación, autorregulación y co-regulación de los aprendizajes, con la finalidad de propiciar el desarrollo de las capacidades del estudiantado enmarcado en un contexto sociohistórico particular, para su formación socioprofesional y finalmente aprender a aprender que es inherente al modelo de EaD.

Tres preguntas clave de la retroalimentación. En algunos estudios y publicaciones se destacan varios tipos de retroalimentación, según su propósito en el proceso de aprendizaje; un ejemplo es lo propuesto por Narciss (2010) citado en Coll, Rochera, Gispert y Díaz (2013) quien señala una retroalimentación para la verificación de información sobre una respuesta y otra para la elaboración la cual "se refiere al conocimiento relativo a los conceptos, requerimientos o reglas de la tarea, errores, cómo proceder o la metacognición, etc." (p.29)

Otro ejemplo es la propuesta de Hirumi (2009) citado en Simonson, Hudgins, y Orellana (2009) quien afirma que, además de que la retroalimentación es de suma importancia en la educación en línea, este debe tener dos formas básicas: confirmatoria y correctiva. La primera le dice al estudiantado qué hicieron correctamente mientras que la segunda brinda recomendaciones de mejora.

Ambos ejemplos dan cuenta de que se pueden encontrar en la literatura e investigaciones diferentes propósitos para la retroalimentación en el proceso educativo; no obstante, requiere del personal docente un proceso reflexivo sobre el porqué y el para qué de su uso, en relación 
con la fundamentación teórico-metodológica de la propuesta educativa y en este caso con la visión de educación a distancia que se tenga.

Una propuesta más amplia para caracterizar la retroalimentación y determinar su función es la de Hattie y Temperley (2007) desde la cual se debe responder a tres preguntas clave: ¿A dónde voy?, ¿cómo voy?, ¿qué sigue?, debido a que a partir de estas se puede definir la ruta de aprendizaje a seguir o lo que se debe modificar a fin de favorecer la construcción de conocimientos. La claridad en la respuesta de cada una de estas interrogantes le va a permitir al estudiantado acercarse más al objeto de conocimiento y lograr alcanzar, en el caso de la educación superior, el perfil socioprofesional esperado.

Así, en primer lugar se requiere tener claro cuáles son las metas u objetivos de aprendizaje, es decir qué se espera que el estudiantado logre. A partir de esto, se deberá prever espacios para la reflexión, autorregulación y para brindar información de retorno; al igual que visualizar la ruta de aprendizaje por seguir. Esto es lo que los autores llaman feed up (Hattie y Temperley, 2007; Alvarado, 2014).

En el caso del diálogo didáctico mediado se esperaría que tanto en los espacios virtuales como en los diversos materiales didácticos se incluyan los propósitos, metas, objetivos con las guías necesarias para que el grupo de estudiantes pueda identificar qué es lo que se espera de cada uno de ellos, no sólo desde lo cognitivo, sino también en lo actitudinal y en lo procedimental; todo esto sin dejar de lado la importancia de conocer cuáles son los propios objetivos y metas de cada estudiante, o al menos propiciar en el diálogo simulado que se da desde los materiales didácticos la reflexión al respecto.

Para tal efecto, es importante que desde el DDM se brinden espacios de diagnóstico y concienciación de las metas u objetivos en función de su utilidad para el aprendizaje, o en el caso de los materiales didácticos, podrían incluir una serie de preguntas o notas insertas (escritas u orales, o las que el tipo de medio permita) que posibiliten al estudiantado determinar cuál es el camino hacia dónde van, a la vez que informe los objetivos que desde el proceso 
educativo se espera que ellos y ellas logren alcanzar; lo cual puede darse al inicio del estudio de cada unidad temática o en diferentes momentos del período lectivo.

Mientras que en los casos donde se realice el DDM sincrónico o anacrónico con uso de la plataforma virtual, se puede realizar un diagnóstico, al iniciar el período lectivo o al iniciar el estudio de las unidades temáticas, para determinar esas metas u objetivos de cada estudiante, así como dar a conocer qué es lo que se espera de su proceso de aprendizaje mediante guías u otro tipo de documento de orientación al estudiantado, de manera que puedan hacer compatibles ambas finalidades; es decir, que más allá de certificar una cantidad de puntos obtenidos, se posea claridad sobre lo que se desea lograr.

Entonces, de esta primer interrogante ¿A dónde voy?, se puede decir que permite evidenciar ese primer acercamiento del estudiantado con el proceso de aprendizaje, ayudándole a ser consciente de la ruta que inicia en su formación dentro de la educación superior, para ello habría que considerar que el diálogo didáctico mediado que se establezca deberá ir de la mano del nivel académico en que se encuentra la población estudiantil y su experiencia previa en la autorregulación de sus aprendizajes.

Posteriormente, a medida que avanza el proceso educativo, es necesario hacer un alto en el camino, las veces que se considere necesario para retomar lo aprendido hasta el momento; por tanto, no se puede decir que existe un momento específico para la pregunta sobre: ¿Cómo voy?, pues tanto el profesorado como el estudiantado podrían formularla. Esta segunda interrogante permitirá generar una reflexión acerca de la construcción de los conocimientos, la cual podría ser respondida de forma individual como grupal, siendo de gran riqueza la interacción de cada estudiante con sus pares y con el profesorado, u otros especialistas en el campo de acción profesional en que se desempeñará el estudiando.

En el caso del diálogo didáctico que se establece en los materiales de cada asignatura en la $\mathrm{EaD}$, es indispensable la inclusión de diferentes opciones para la autoevaluación, que no sean simplemente un cuestionario de preguntas y respuestas, sino que favorezcan en el 
estudiantado el desarrollo de su capacidad de analizar así como de valorar cómo ha sido su aprendizaje, cuáles han sido las estrategias de aprendizaje que les han funcionado y cuáles no, cuál ha sido su nivel de avance y qué requiere mejorar.

Adicionalmente, es fundamental el acompañamiento que brinde el profesorado durante este proceso, es decir, no se puede dejar todo el proceso al estudiante, si bien es cierto, el material didáctico debe permitir la autonomía del aprendizaje, esto no significa que la orientación docente no sea necesaria, por el contrario, debe existir la disponibilidad para brindar esa información de retorno que le permita hacer las modificaciones del caso. Situación que debe ser mucho más evidente en la formación que hace uso de las plataformas virtuales para el aprendizaje, pues permite una mejor y mayor interacción entre la figura docente, el estudiantado y sus pares.

Como tercera interrogante surge el ¿qué sigue?, es decir qué acciones debe realizar el estudiantado a partir de la reflexión acerca de sus progresos o de las mejoras por implementar en el proceso de aprendizaje. La respuesta dada debe orientarse a la formulación de nuevos retos, así como a generar oportunidades de mejora en la autorregulación, de manera que pueda retomar o modificar la ruta seguida para alcanzar las metas u objetivos previstos.

Podría decirse que este es quizá el momento de retroalimentación más valioso, pues permite evidenciar la comprensión profunda de cada estudiante sobre el objeto de conocimiento, así como el desarrollo de su proceso metacognitivo; lo cual constituye un reto importante dentro de la EaD, puesto que en el diálogo didáctico deberá contemplarse una serie de orientaciones o guías que permitan, en la interacción sincrónica o anacrónica, favorecer en el estudiantado el planteamiento de mejores estrategias o el uso de otras herramientas para su aprendizaje, que no sólo sean útiles para un momento específico sino que sean transferibles a otras situaciones. Esto es lo que los autores llaman feed forward (Hattie y Temperley, 2007) o proalimentación (Rodríguez e Ibarra, 2011) 
Por otra parte, dentro de este modelo de retroalimentación planteado por Hattie y Temperley (2007), los autores añaden que cada una de estas preguntas deben ser respondidas en cuatro niveles, a saber: a nivel de la tarea de aprendizaje (¿cómo está siendo comprendida y ejecutada la tarea asignada?); a nivel del proceso de aprendizaje (¿cómo está siendo comprendido y ejecutado el proceso de aprendizaje?); a nivel de autorregulación (¿cuáles son las estrategias o acciones de autorregulación y regulación implementadas?); así como a nivel de la persona como tal (¿cuáles son las evaluaciones que hace sobre la persona?), siendo esta última la que menos se utiliza por el cuidado que habría que tener para que la retroalimentación se centre más en los procesos de aprendizaje y desempeños que en la persona en sí.

A partir del planteamiento expuesto, se puede decir que la retroalimentación no puede ser considerada como un momento específico dentro de las acciones educativas que se emprendan, que solo atiende a un producto o desempeño estudiantil para obtener un puntaje dentro de la calificación establecida. En el DDM la retroalimentación debe ser un proceso continuo que brinde el acompañamiento y orientación necesaria para cada estudiante; para ello, se debe hacer uso de todos los recursos disponibles para la interacción y desde una visión multidireccional en la comunicación.

Al respecto, Alvarado (2014) menciona

La retroalimentación es fundamental, pues es a través de ésta que el alumno no solo se da cuenta de sus fortalezas y debilidades, sino que es una oportunidad que el profesor tiene para promover la reflexión en el aprendizaje y construir un continuo durante todo el curso y generar así, un conocimiento aún más profundo y significativo (p.70).

Principios de la retroalimentación. Wiggins (2012) apunta a siete principios que el autor ha llamado "las siete llaves de para una retroalimentación efectiva", estas son: referido a una meta, tangible y transparente, realizable, amigable para el usuario, a tiempo, en el proceso así como consistente. 
Según este autor, la retroalimentación debe darse en el momento que sea requerido, pues cuando se realiza de forma tardía el estudiantado tiene dificultades en las tareas o procesos posteriores. Esto constituiría uno de los desafíos en el DDM que se realiza a partir de los materiales didácticos, pues al ser un diálogo simulado, no puede darse en aislado; para ello es fundamental el contacto con el profesorado, ya sea a través del uso de las herramientas de interacción de la plataforma virtual y de otros medios de comunicación, los que serán de importancia para dar la retroalimentación oportuna, siempre y cuando se dé el seguimiento requerido al estudiantado.

Asimismo, la transparencia y lo tangible de la retroalimentación se garantiza en la medida en que la información que se brinde esté relacionada con los objetivos y metas establecidos; para esto se debe procurar que en el intercambio de información sobre la tarea o los procesos, la persona tenga claro qué es lo que debe mejorar y genere las estrategias sobre cómo hacerlo. Quizá en las primeras experiencias del estudiantado en el sistema de $\mathrm{EaD}$, se requiera brindar un mayor acompañamiento y guía por parte del profesorado, a fin de favorecer el desarrollo de las estrategias metacognitivas que le permitan autorregularse.

De igual manera, para la retroalimentación se puede recurrir a la colaboración entre pares, que va a propiciar en todo caso la co-regulación. Sin embargo, debe tomarse en cuenta que esto requiere de estudiantes con un mayor dominio de los procesos reflexivos, críticos y metacognitivos.

Por otra parte, Nicol y MacFarlane-Dick citados en Gilbert, Whitelock y Gale (2011) identifican siete principios de la retroalimentación:

1. Ayuda a clarificar qué es un buen desempeño (metas, criterios, estándares esperados)

2. Facilita el desarrollo de la autoevaluación (reflexión) del aprendizaje.

3. Provee al estudiantado información de alta calidad acerca de su aprendizaje

4. Fomenta el diálogo entre pares y con el profesorado sobre el aprendizaje.

5. Fomenta la autoestima y las creencias motivacionales positivas. 
6. Provee oportunidades para disminuir la brecha entre el desempeño actual y el deseado.

7. Brinda información al profesorado que puede ser usada en la forma de enseñar. (pp.34-35) (traducción)

De igual manera, Gibbs en Brown y Glasner (2010) retoma de los principios de Chickering y Gamso para una buena práctica de la educación, que destaca sobre la retroalimentación que "ésta debe darse en un tiempo breve posterior a la actividad de aprendizaje" (p.66). Al respecto, el autor reconoce que cuando el grupo de estudiantes es numeroso esto puede dificultarse, pero señala que la utilidad e importancia que vea el estudiantado en la información proporcionada sobre su actuación o producto es muy valioso; de igual manera, considera relevante el trato personalizado a la hora de realizar la retroalimentación, y la socialización de productos o actuaciones con sus pares permite que cada estudiante dé mayor valor e importancia a la retroalimentación.

Por otro lado, Evans (2013) señala como principios de la práctica de retroalimentación los siguientes:

- La retroalimentación se da durante el proceso de evaluación y es parte integral de éste.

- La orientación desde la retroalimentación es explícita.

- Da mayor énfasis en la proalimentación que a la retroalimentación.

- El estudiantado está comprometido en y con el proceso.

- Los detalles técnicos de la retroalimentación son atendidos con la finalidad de apoyar el aprendizaje.

- La capacitación en proalimentación y retroalimentación es parte integral del diseño de la evaluación (traducción) pp.80-83).

Cada uno de estos principios, como se puede ver, son aplicables al DDM partiendo que éste promueve el uso de multiplicidad de recursos y formas de interacción que propician, 
precisamente, el intercambio y el diálogo entre los diferentes agentes que participan del proceso educativo para la construcción del conocimiento.

En síntesis, se propone que desde el DDM se consideren al menos los siguientes principios básicos de la retroalimentación:

- Debe ser parte integral del proceso de aprendizaje.

- Debe darse a partir de la interacción multidireccional, con el acompañamiento del profesorado.

- Se debe brindar en el momento oportuno, haciendo uso de diversos medios para la comunicación entre profesorado y estudiantado.

- Debe propiciar la autonomía, la reflexión y autorregulación del aprendizaje, así como la transferencia de conocimientos.

- Debe ser concordante con los objetivos y metas de aprendizaje.

- Debe permitir dar respuesta a las tres preguntas clave: ¿a dónde voy? ¿cómo voy? ¿qué sigue?

\section{Conclusiones}

A partir de lo anteriormente expuesto se puede concluir:

- La interacción en la $\mathrm{EaD}$ es un componente esencial que debe propiciar la retroalimentación, en este sentido no puede ser improvisada ni tampoco obviarse; para ello es necesario hacer uso de todos los recursos disponibles a fin de dar una retroalimentación oportuna, útil y válida.

- La retroalimentación debe ser un proceso intrínseco a la enseñanza y al aprendizaje; por tanto, se debe tener previsto su incorporación tanto en los materiales didácticos como en las otras formas de interacción que se propongan al estudiantado.

- La retroalimentación implica no sólo aquella información que se da a cada estudiante, sino la información que el estudiantado brinda al profesorado o a sus pares para el mejoramiento del proceso de enseñanza y de aprendizaje.

- Los materiales didácticos para la EaD deben ser producidos de tal manera que permitan orientar al estudiante no sólo en su proceso de construcción de conocimientos, sino que 
le permita reflexionar sobre su aprendizaje, pero estos deben ser complementados con la interacción con el profesorado, ya sea cara a cara o a través las herramientas de comunicación disponibles.

- La implementación de la retroalimentación desde el DDM requiere que el personal que interviene en la docencia tenga la formación para poder generar una interacción que brinde información oportuna, útil y significativa para el proceso de aprendizaje del estudiantado. Esto significa que el equipo que participa en la producción de los materiales así como en el diseño del entorno virtual debe poseer la formación en los procesos de mediación pedagógica, regulación y autorregulación.

- Se requiere brindar formación al estudiantado en procesos de reflexión, autorregulación y autoevaluación del aprendizaje, considerando un mayor acompañamiento a quienes no han tenido experiencia de estudio en EaD.

En consecuencia, es recomendable realizar procesos de investigación acerca de las mejores estrategias para la retroalimentación desde los materiales didácticos; de igual forma, se puede ahondar también en los procesos de retroalimentación que realiza el grupo de estudiantes entre sí y cómo desde la docencia orientar este proceso; al igual que los efectos de la retroalimentación en la mejora de los procesos de enseñanza a distancia.

\section{Referencias}

Alvarado, M. (2014) Retroalimentación en Educación en línea: una estrategia para la construcción del conocimiento. Revista iberoamericana de educación a distancia, RIED 17(2), 59-73.

Anderson, T. (2008) (Edit.). The theory and practice of online learning. Edmonton: Athabasca University Governing Council.

Anderson, T. y Dron, J. (marzo, 2011) Three generations of distance education pedagogy. IRROLD International review of research in open and distributed learning, 12(3), 80-97. Recuperado de http://www.irrodl.org/index.php/irrodl/article/view/890/1826

Brown, S. y Glasner, A. (Edits.) (2010) Evaluar en la Universidad. Problemas y nuevos enfoques. Madrid, España: Narcea. 
Coll, C., Rochera, M. J., De Gispert, I., y Diaz, F. (2013). Distribution of Feedback among Teacher and Students in Online Collaborative Learning in Small Groups. Digital Education Review, 23, 27-46.

Evans, C. (marzo, 2013). Making sense of assessment feedback in higher education. Review of educational research, 83(1), 70-120. Doi: 10.3102/0034654312474350.

Ferguson, P. (2011). Student perceptions of quality feedback in teacher education. Assessment \& evaluation in Higher Education, 36(1), 51-62. Doi: 10.1080/02602930903197883

García, L. (2014) Bases, mediaciones y futuro de la educación a distancia en la sociedad digital. Madrid, España: Editorial Síntesis.

Gilbert, L., Whitelock, D. y Gale, V. (Julio, 2011) Synthesis report on assessment and feedback with technology enhancement. Learning societies lab in the School of Electronics and Computing Sciences, University of Southampton y Institute of Educational Technology, The Open University. Recuperado de http://eprints.soton.ac.uk/273221/1/Synthesis\%20report\%20on\%20assessment\%20an d\%20feedback\%20Final\%20Report\%20July\%202011.pdf

González, P. (1988) La conversación didáctica guiada en la elaboración de material instruccional para la educación a distancia. Informe de investigaciones educativas, II (2), 201-218. Recuperado de http://biblo.una.edu.ve/ojs/index.php/llE/article/view/188/178

Gutiérrez, F y Prieto, D. (1999). La mediación pedagógica. Apuntes para una educación a distancia alternativa. Argentina: Ediciones La Crujía.

Hattie, J. y Timperley, H. (marzo, 2007). The power of feedback. Review of Educational Research, 77(1), 81-112. DOI: 10.3102/003465430298487

Holmberg, B., Shelley, M., y White, C. (2005). Distance Education and Languages: Evolution and Change. Clevedon: Multilingual Matters.

Lozano, F.G. y Tamez, L. A. (2014). Retroalimentación formativa para estudiantes de la educación a distancia. RIED. Revista Iberoamericana de Educación a Distancia, 17(2), 197-221. Recuperado de http://www.redalyc.org/articulo.oa?id=331431248010

Medel-Añonuevo, C., Ohsako, T. y Mauch, W. (2001) Revisiting lifelong learning for the 21st century. Filipinas: UNESCO Institute for Education. Recuperado de http://www.unesco.org/education/uie/pdf/revisitingLLL.pdf

Moreno, M. (Coord.) (2012) Veinte visiones de la educación a distancia. Guadalajara, México: UDGVIRTUAL.

Recuperado

de file:///G:/Users/nsalas/Documents/Proyecto\%20Evaluaci\%C3\%B3n\%20Aprendizajes/2 0 visiones educaci\%C3\%B3n distancia.pdf 
Rodríguez, G. \& Ibarra, S. (Edits.) (2011) e-Evaluación orientada al e-aprendizaje estratégico en educación superior. Madrid, España: Narcea.

Rodríguez, P. (enero-febrero, 2007) Un modelo de comunicación en la educación a distancia. Una reflexión educomunicativa. Innovación educativa, 7(36), 36-51. Recuperado de http://www.redalyc.org/pdf/1794/179420814004.pdf

Sanmartí, N. (2007) Diez ideas clave. Evaluar para aprender. Barcelona, España: Editorial Grao.

Simonson, M. R., Hudgins, T. L., \& Orellana, A. (2009). The Perfect Online Course: Best Practices for Designing and Teaching. Charlotte, N.C.: Information Age Publishing.

Simonson, M., Smaldino, S., Albright, M. \& Zvacek, S. (2009) Teaching and learning at a Distance. Foundations of distance education. (4a. Ed.) USA: Pearson Education.

Wiggins, G. (setiembre, 2012). Seven Keys to Effective Feedback. Educational Leadership, 70(1), 10-16. Recuperado de http://web.b.ebscohost.com.talamanca.uned.ac.cr/ehost/pdfviewer/pdfviewer?vid=6\&si

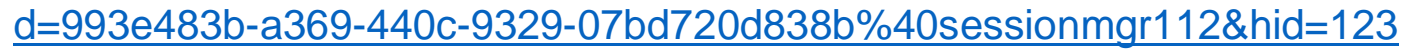

